\title{
A MODEL OF INTERDEPENDENT INFRASTRUCTURE SYSTEM RESILIENCE
}

\author{
JINGJING KONG \& SLOBODAN P. SIMONOVIC \\ Department of Civil and Environmental Engineering, Western University, London, ON, Canada.
}

\begin{abstract}
Infrastructure systems of transportation, water supply, telecommunications, power supply, etc. are not isolated but highly interconnected and mutually coupled. Infrastructure interdependences can increase system vulnerability and produce cascading failures at the regional or national scales. Taking the advantage of network theory structure analysis, this paper models a multilayer infrastructure network of street, water supply, power supply and information infrastructure layers. The infrastructure interdependences are detailed using five basic dependence patterns of basic network elements. Definitions of dynamic cascading failures and recovery mechanisms of infrastructure systems are also established. The main focus of the paper is the introduction of a dynamic measure of infrastructure network resilience capable of addressing infrastructure system, as well as network component (layer), interdependences. The measure is based on infrastructure network performance, proactive infrastructure network resistance capacity and reactive infrastructure network recovery capacity. With three resilience features and corresponding network properties, this paper develops the quantitative measure of dynamic space-time resilience and a resilience simulation model for infrastructure networks. The resilience model is applicable to any type of infrastructure and its application can improve the decision-making processes of infrastructure planning, design and maintenance.
\end{abstract}

Keywords: infrastructure interdependence, infrastructure system, multilayer network, resilience.

\section{INTRODUCTION}

Due to the rising cost of infrastructure upkeep and increasing frequency of extreme events affecting its functioning, Canada's infrastructure systems have become more vulnerable to natural disasters. Recent examples include the Alberta and Toronto floods of 2013. Infrastructure systems consist of diverse infrastructure elements, including telecommunications, power supply, natural gas and oil, transportation, water supply, etc. Interdependencies among different infrastructure elements can produce cascading failures throughout the whole infrastructure system at regional and national scales [1]. So the infrastructure system resilience is often overestimated [2] and the corresponding protection and recovery strategies don't always provide the desired results.

Infrastructure system resilience refers to the ability of system to resist possible disturbance, absorb the initial damage, and recover to normal operation [3, 4]. The Multidisciplinary Center for Earthquake Engineering Research (MCEER) provides a general resilience framework for definition and quantification of the physical and organizational systems resilience to earthquakes [5]. As a follow up of this work, many studies emerged on the quantification of performance and resilience assessment of a utility system with its operational mechanism, such as water supply system [6], electric infrastructure system [7], telecommunications cable system [8], underground transportation [9], natural gas network [10] among others. Some of the reported research focuses on resilience assessment of interdependent infrastructure systems using input-output inoperability model [11, 12].

Taking the advantage of network theory structure analysis, infrastructure systems can be described as complex networks, where nodes represent infrastructure components (such as water pumps, electric transformers, and similar), and links mimic the physical and relational 
connections among different infrastructure components (such as electric tie lines, water pipes etc.) [13]. Cascading failures across diverse infrastructure systems can be simulated using topology-based or flow-based methods that lead to the estimation of multi-infrastructure system vulnerability. Some of the published research is clearly emphasising recovery processes to evaluate for example, gas and electric infrastructure system resilience [14, 15]. However, the infrastructure system resilience should address both, proactive adaptive capacity and reactive recovery capacity.

Simonovic and Peck [16] point out that continued operation and rapid restoration of the systems affected by a disturbance are essential for resilience. A resilient infrastructure system is a sustainable network of critical lifelines that "possess the capacity to survive, cope, recover, learn and transform from disturbances". So based on the Space-Time Dynamic Resilience Measure of Simonovic and Peck [16], this paper integrates multi-infrastructure network properties and defines an infrastructure system resilience model for quantifying both, dynamic proactive adaptive capacity and reactive recovery capacity with three dimensions of resilience and network properties.

The remainder of this article is organized as follows. Section 2 presents the infrastructure system model, including street, water supply, power supply and information infrastructure components, as a network of networks, or a multilayer infrastructure network model. Basic dependence patterns of individual infrastructure components for establishing system dynamic cascading failures and recovery mechanisms are also provided. Section 3 provides a definition of a multilayer infrastructure network resilience, and presents dynamic resilience metric under sequential disturbances. Finally, the potential resilience model applications are discussed in Section 4.

\section{INFRASTRUCTURE NETWORK FORMALIZATION}

\subsection{Infrastructure Network Representation}

The infrastructure network model proposed here is based on the network theory, where two basic components, nodes and edges, are used to build the model of a system. A network is always represented by $G$, the nodes set and edges set are represented by $N$ and $E$ respectively. This paper focuses on the main urban infrastructure system networks including streets, power grid, water supply, and information infrastructure.

A street network is represented as $G^{S}\left(N^{S}, E^{S}\right)$, where $N^{S}$ is the set of street junctions and end points, and $E^{S}$ is the set of street segments [17]. The edges are undirected and homogeneous. Generally, the street network is fully connected. A water supply network is represented as $G^{W}\left(N^{W}, E^{W}\right)$, where waterworks, storage facilities and pump stations are represented as nodes with different attributes, and water distribution pipes are describes as edges [18]. The edges of the water supply network are directed as the water flow from waterworks to pump stations and storage facilities through distribution pipes. Generally, water supply networks are represented as trees without circular and redundant edges. The downstream nodes and edges could not operate unless all the upstream nodes and edges function normally. A power grid is represented as $G^{P}\left(N^{P}, E^{P}\right)$, where power plants, distribution and transmission substations are represented by nodes with different attributes, and power lines are represented as directed edges [19]. Same as for water supply networks, the edges of a power grid are directed as the electricity is transmitted from power plants to transmission substations, and then to distributing stations through power lines. The downstream nodes and edges could not operate 
unless all the upstream nodes and edges function normally. Information infrastructure is represented as $G^{I}\left(N^{I}, E^{I}\right)$, where the Internet service providers are represented as nodes, and cable connections as undirected edges [8]. Since these networks provide bidirectional exchange of information, the edges are undirected. According to scale, population and structure of a city, information network structure could be represented using a star, chain or circular shape, and so on. A node or edge operate normally if there is an existing path connecting to the source node.

All individual infrastructure networks introduced above can be illustrated as individual infrastructure layers. An infrastructure system model is a network of networks integrating all of the layers, as illustrated in Fig. 1. Nodes and edges in the same layer belong to the same kind of infrastructure (intra-infrastructure connection, which is denoted by solid lines within the single layer networks in Fig. 1). Edges between different layers describe dependences and connections between different types of infrastructure (inter-infrastructure connections, which are denoted by dotted lines connecting different layer networks in Fig. 1). The red dotted lines between nodes that belong to the power grid and the water supply network represent electricity provided from the electric infrastructure to water supply infrastructures.

As different infrastructure components located in the same area are subject to a specific disturbance (disaster), it is necessary to consider the location of infrastructure in the model description. Furthermore, the location of infrastructure has important effect on topological properties and consequently on infrastructure functioning processes. So, the spatial attributes of nodes and edges should be included in a realistic infrastructure network model with geographic coordinates, which can be defined in a two-dimensional Euclidean coordinate system. Therefore, each node has three coordinates $(\phi, x, y)$, where $\phi$ denotes the type of infrastructure, $(x, y)$ denotes the geographic location of the node. Edges are scribed by the two adjacent nodes.

\subsection{Basic Infrastructure Dependence Patterns}

The interdependent networks represent complex systems where emergent behaviors are rarely fully understood. Urban infrastructure components can be dependent and interdependent in

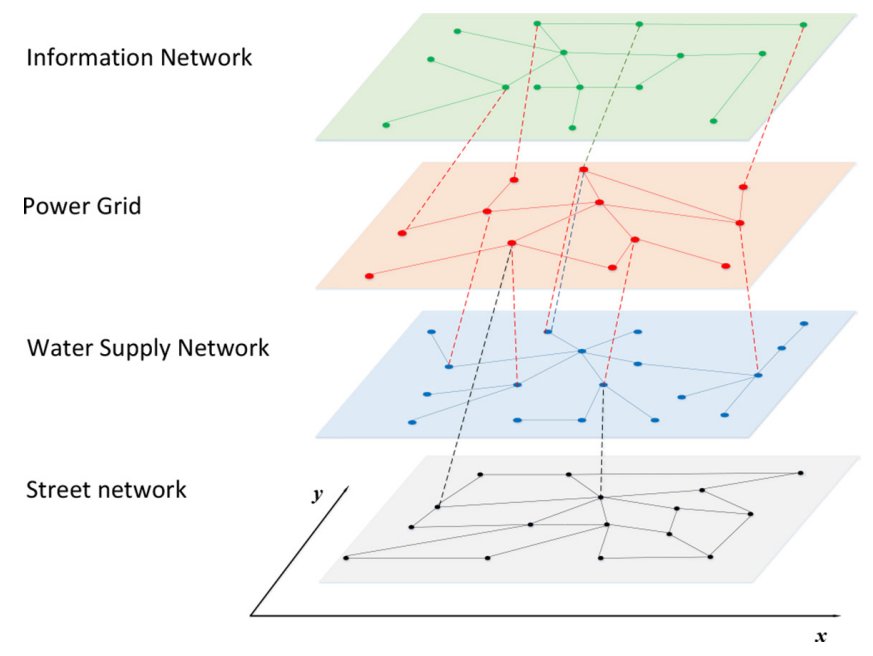

Figure 1: Interdependent infrastructure system model representation. 
various ways. Most of the earlier literature view interdependencies as macro-properties of coupled systems classified in different ways. For example, Dudenhoeffer et al. [20] classify interdependences into four types: physical, geospatial, policy and informational.

Interdependence indicates the bidirectional interaction, which includes two directed dependences between two infrastructure elements. Generally, any components malfunction of one infrastructure system can result in efficiency reduction, function loss or system destruction of another macro-interdependent infrastructure system. So, the macro-interdependence is a function of the system attributes and state of the malfunction infrastructure systems. Therefore, the micro structure, or the basic pattern of infrastructure dependence, needs to be considered. The focus of the proposed resilience model is a direct impact of infrastructure malfunction, which is always seen as the first-order effect.

Let us consider an infrastructure system of two network layers $G^{\phi_{1}}$ and $G^{\phi_{2}}$ where $\phi_{1} \neq \phi_{2}$. Every element of these two networks has two exclusive states: on (normal operation) and off, (non-operation and malfunction). Normal operation refers to the designed level of operation. Non-operation indicates physical destruction of elements by a disturbance, and malfunction describes the situation when elements are not physically destroyed but could not function due to related resources outage. With different relations between four fundamental network structure elements, nodes, edges, paths and clusters (combinations of nodes and edges), there are five basic infrastructure dependence patterns (four patterns are illustrated in Fig. 2):

1. Node-node dependence (Fig. 2a): the state of node $n_{i}^{\phi_{1}}$ is dependent on the state of $n_{j}^{\phi_{2}}$ via resource, service and information flows between the nodes. For example, the state of a water pump depends on the state of its connecting electric transmission substation. This pattern is represented as:

$$
I D^{n n}=e_{i j}^{\phi_{1} \phi_{2}}
$$

where $I D^{n n}$ is node to node dependence between two networks, and $e_{i j}^{\phi_{1} \phi_{2}}$ is state of the edge linking two nodes: node $i$ of $\phi_{1}$ network and node $j$ of $\phi_{2}$ network.

(i) Node-edge dependence (Fig. 2b): the state of node $n_{l}^{\phi_{1}}$ is dependent on the edge $e_{i j}^{\phi_{2}}$ or $\left(n_{i}^{\phi_{2}}, n_{j}^{\phi_{2}}\right)$, or vice versa. For example, the state of Internet service provider depends on its connecting power supply. This pattern is represented as:

$$
I D^{n e}=n_{l}^{\phi_{1}} \times e_{i j}^{\phi_{2}}
$$

where $I D^{n e}$ is node edge dependence, or vice versa. $n_{l}^{\phi_{1}}$ is the state of node $l$ of $\phi_{1}$ network, and $e_{i j}^{\phi_{2}}$ is the state of edge of $\phi_{2}$ network linking nodes $i$ and $j$.

2. Node/Edge - path dependence (Fig. 2c): the state of node $n_{l}^{\phi_{1}}$ or edge $e_{l k}^{\phi_{1}}$ is dependent on the state of the path $p_{i m}^{\phi_{2}}$, which is represented as $\left\{n_{i}^{\phi_{2}}, e_{i j}^{\phi_{2}}, n_{j}^{\phi_{2}}, e_{j k}^{\phi_{2}}, n_{k}^{\phi_{2}}, \ldots, n_{m}^{\phi_{2}}\right\}$. For example, the state of coal power plant is dependent on the path (transportation network) connecting the plant with coal supply locations. This pattern is represented as

$$
I D^{N P}=n_{l}^{\phi_{1}} \times p_{i m}^{\phi_{2}} \quad \text { or } \quad I D^{E P}=e_{l k}^{\phi_{1}} \times p_{i m}^{\phi_{2}}
$$

where $I D^{N P}$ is node path dependence $n_{l}^{\phi_{1}}$ is the state of node $l$ of $\phi_{1}$ network, and $p_{i m}^{\phi_{2}}$ is the state of path connecting node $i$ and node $m$ of $\phi_{2}$ network, and $p_{i m}^{\phi_{2}}=\prod_{i}^{m} n_{i}^{\phi_{2}} \prod_{i, j}^{, m} e_{i, j}^{\phi_{2}} . I D^{E P}$ is edge another network path dependence, $e_{l k}^{\phi_{1}}$ is the state of edge linking nodes $l$ and $k$ of $\phi_{1}$ network. 


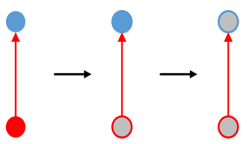

(a) Node-node dependence

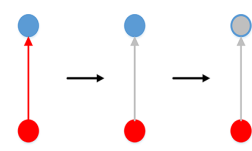

(b) Node-node dependence

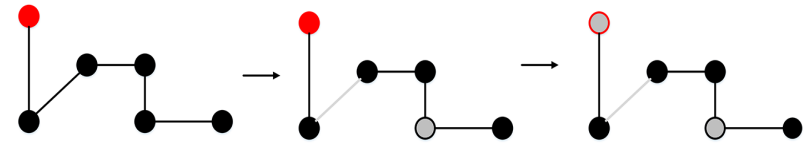

(c) Node-path dependence

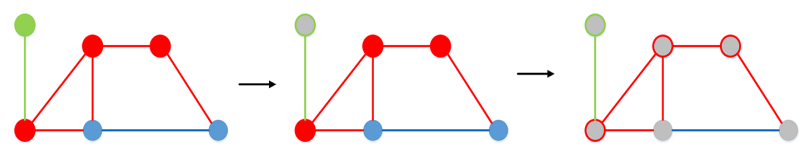

(d) Node-cluster dependence

Figure 2. Basic infrastructure dependence patterns.

(Nodes and edges in blue, red, black and green represent water, electric, street and communication infrastructure networks respectively; grey inside represent the malfunction state of the infrastructures; arrows represent the change over time.)

3. Node/Edge - cluster dependence (Fig. 2d): the state of cluster $c_{i}^{\phi_{1}}$, which is a set of nodes and their edges of network $G^{\phi_{1}}$, is dependent on the state of node $n_{l}^{\phi_{2}}$ or edge $e_{l k}^{\phi_{2}}$ of network $G^{\phi_{2}}$. For example, the operations of water or power infrastructure with the same geographic attributes being controlled by an internet service provider. This pattern is represented as:

$$
I D^{N C}=n_{l}^{\phi_{2}} \times c_{i}^{\phi_{1}} \quad \text { or } \quad I D^{E C}=e_{l k}^{\phi_{2}} \times c_{i}^{\phi_{1}}
$$

where $I D^{N C}$ is cluster node dependence. $n_{l}^{\phi_{2}}$ is the state of node $l$ of $\phi_{2}$ network, and $c_{i}^{\phi_{1}}$ is the state of cluster of $\phi_{1}$ network. $I D^{E C}$ is cluster network edge dependence, $e_{l k}^{\phi_{2}}$ is the state of edge linking nodes $l$ and $k$ of $\phi_{2}$ network. $n_{i}^{\phi_{1}}$ and $e_{i j}^{\phi_{1}}$ are the state of elements of the cluster $c_{i}^{\phi_{1}}$, and $c_{i}^{\phi_{1}}=\prod n_{i}^{\phi_{1}} e_{i j}^{\phi_{1}},\left(n_{i}^{\phi_{1}} \in c_{i}^{\phi_{1}}, e_{i j}^{\phi_{1}} \in c_{i}^{\phi_{1}}\right)$.

4. Geographic dependence: the state of all infrastructure elements located at the same location $A$ are affected by a disturbance simultaneously. This pattern is represented as:

$$
I D^{G L}=\left\{n_{i}^{\phi_{1}} \cup e_{j k}^{\phi_{1}} \cup \cdots \cup n_{p}^{\phi_{1}} \cup e_{q r}^{\phi_{1}}\right\}
$$

where $I D^{G L}$ is geographic dependence among nodes and edges. $n_{i}^{\phi_{1}}, e_{j k}^{\phi_{1}}, \ldots, n_{p}^{\phi_{1}}, e_{q r}^{\phi_{1}}$ are nodes and edges with coordinate values $(x, y)$ belonging to the same area $\mathrm{A}$.

In the previous discussion we looked at two infrastructure network system. The basic dependence patterns can cause cascading impacts throughout the multilayer network as time goes on. Given three individual infrastructure networks $G^{\phi_{1}}, G^{\phi_{2}}, G^{\phi_{3}}\left(\phi_{1} \neq \phi_{2} \neq \phi_{3}\right)$, there are many combinations of the five basic dependence patterns, which could form a chain or cycle reaction among three single layer network system and cause cascading failure spreading throughout the whole infrastructure system. On the other hand, interdependences could accelerate mitigation and be conducive to disturbance response with repair of several components. They can contribute to strengthening system robustness and resilience with local protection. 


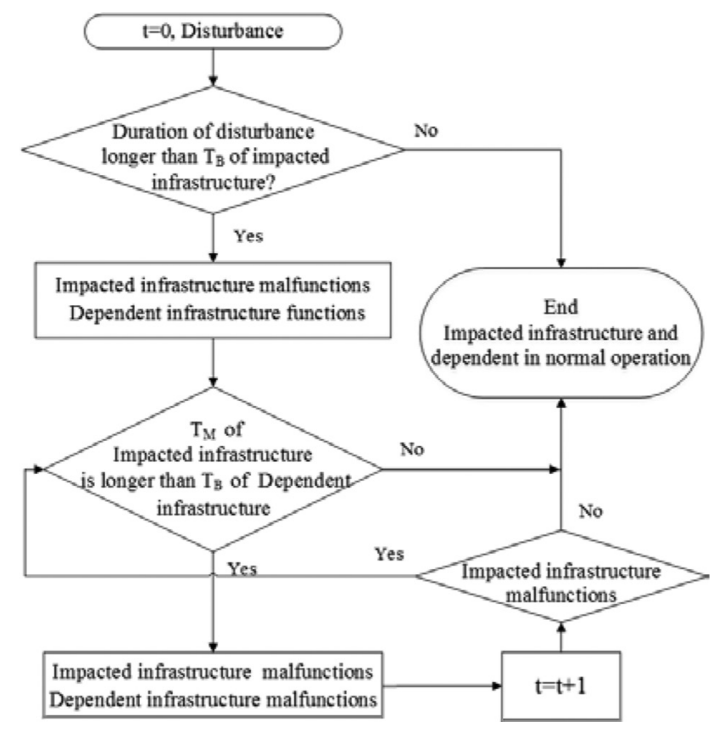

Figure 3: Dynamic description of infrastructure system performance.

\subsection{Infrastructure System Dynamic Mechanism}

Magnitude of the interrupted service and duration of the interruption are the two main disturbance characteristics for the assessment of consequences [13]. In practice, an infrastructure could: (i) absorb the impacts of disturbance and minimize consequences with little effort (i.e. buffering); (ii) adjust to undesirable conditions by undergoing some changes (adaptation); and (iii) fully recover from disturbance. All three response modes define the infrastructure adaptive capacity [4]. The response of the infrastructure system to a disturbance varies with time - adding dynamic properties to interdependent infrastructure networks [14].

In order to capture the dynamic character of the disturbance consequences, the change of the infrastructure performance due to various disturbances needs to be estimated. As discussed in Section 2.2, the state of an infrastructure is influenced by the disturbance as well as the state of other connected infrastructure. Let $T_{B}$ denote buffering time of an infrastructure system, $T_{R}$ its repair time; and $T_{M}$ its malfunction time. The dynamic performance of an infrastructure system subject to a disturbance can be illustrated using the flow chart in Fig. 3. Infrastructure system in the illustration can be a node, an edge, a cluster and a path of an infrastructure network. Its state is decided by corresponding basic dependence patterns, and calculated using expressions (1)-(5).

\section{INFRASTRUCTURE SYSTEM RESILIENCE MODEL}

\subsection{Infrastructure System Resilience Definition}

The infrastructure system resilience is defined as "the ability to prepare for, and adapt to changing conditions and withstand and recover rapidly from disruptions", including "the ability to withstand and recover from deliberate attacks, accidents, or naturally occurring threats or incidents" [15]. So, infrastructure system resilience is defined from the system performance and its adaptive capacity that can be in two different forms: proactive 
adjustment capacity and reactive recovery capacity. As the number of functioning infrastructure elements and the amount of resources left are the foundations for recovery, the former capacity will directly influence the latter.

An infrastructure system, a typical "systems of systems", is a set of multiple and independently operational systems interacting with one another to meet specific needs. Therefore, infrastructure system resilience refers not only to the ability to resist disturbance and reorganize while undergoing change of intra-layer networks, but also the ability to retain essentially the same function, structure and feedbacks among inter-layer networks. The former capacity relates with an individual infrastructure system. The latter needs more systematic thinking and management due to potentially small unforeseen disturbances.

\subsection{Three Features of Infrastructure System Resilience}

System resilience can be represented and quantified by four key features: robustness, redundancy, resourcefulness and rapidity [5]. As proactive adjustment capacity is a function of network robustness, reactive recovery capacity can be represented by network resourcefulness and rapidity. Redundancy can be seen as the cause of robustness and therefore in this paper we analyze the three key features of resilience: robustness, resourcefulness and rapidity.

\subsubsection{Robustness}

Robustness refers to the ability of a system to withstand a given level of stress without suffering degradation or loss function. The common measure for network robustness is the critical fraction at which the system completely collapses. Similarly, for individual infrastructure, the robustness is computed as the ratio of minimum number of operational elements after a disturbance $\zeta_{1}$ to the total number of elements of one infrastructure type (one network layer), and repressed as:

$$
R_{R o b}^{\phi, \zeta_{1}}\left(t_{1 R}^{\phi}\right)=\frac{n_{o}^{\phi}\left(t_{1 R}^{\phi}\right)+e_{o}^{\phi}\left(t_{1 R}^{\phi}\right)}{N^{\phi}+E^{\phi}}
$$

where $t_{1 R}^{\phi}$ is the time when the sum of $n_{o}^{\phi}(t)$ and $e_{o}^{\phi}(t)$ generates the maximum loss of a system $\phi$ performance after the disturbance $\zeta_{1}, n_{o}^{\phi}$ is the number of operational nodes and $e_{o}^{\phi}$ is the number of operational edges, $N^{\phi}$ and $E^{\phi}$ are the total numbers of nodes and edges, respectively.

For a multilayer infrastructure network, the robustness is computed as the ratio of the minimum number of operational elements to the total number of elements over all infrastructure network types (layers), which is shown in Fig. 4 and expressed as:

$$
R_{R o b}^{\zeta_{1}}\left(t_{1 R}\right)=\frac{\sum_{\phi}\left(n_{o}^{\phi}\left(t_{1 R}\right)+e_{o}^{\phi}\left(t_{1 R}\right)\right)}{\sum_{\phi}\left(N^{\phi}+E^{\phi}\right)}
$$

where $t_{1 R}$ is the time when the sum of $n_{o}^{\phi}\left(t_{1 R}\right)$ and $e_{o}^{\phi}\left(t_{1 R}\right)$ of all single layer networks generates the maximum loss of performance after the disturbance $\zeta_{1}, n_{o}^{\phi}\left(t_{1 R}\right)$ is the number of operational nodes and $e_{o}^{\phi}\left(t_{1 R}\right)$ is the number of operational edges of a single layer network $\phi$ at $t_{1 R}$, $N^{\phi}$ and $E^{\phi}$ are the total numbers of nodes and edges of single layer network $\phi$, respectively. 


\subsubsection{Resourcefulness}

Resourcefulness is the capacity to develop and implement mitigation and response strategies to a specific disturbance. It is limited by the ability to obtain sufficient monetary, physical, technological, informational and human resources necessary to meet established priorities. In this work, the network performance of restoration strategies to a specific disturbance $\zeta_{1}$ is used for quantifying resourcefulness, expressed as:

$$
R_{\mathrm{Re} s}^{\phi, \zeta_{1}}(t)=S P^{\phi, \zeta_{1}}(t)-S P_{0}^{\phi, \zeta_{1}}(t)=f\left(S P^{\phi, \zeta_{1}}(t-1), R S^{\phi, \zeta_{1}}(t-1)\right)-S P_{0}^{\phi, \zeta_{1}}(t)
$$

where $S P^{\phi, \zeta_{1}}(t)$ is the system performance of network $\phi$ with restoration starting at $t, S P_{0}^{\phi, \zeta_{1}}(t)$ is the system performance of network $\phi$ without restoration at $t, R S^{\phi, \zeta_{1}}(t-1)$ is the response strategy of infrastructure network $\phi$ after disturbance $\zeta_{1}$ occurence at $t-1 . f(\bullet)$ is the result of $S P^{\phi, \zeta_{1}}(t-1)$ and $R S^{\phi, \zeta_{1}}(t-1)$.

For multilayer infrastructure network, resourcefulness is quantified as:

$$
R_{R e s}^{\zeta_{1}}(t)=S P^{\zeta_{1}}(t)-S P_{0}^{\zeta_{1}}(t)=F\left(\sum S P^{\phi, \zeta_{1}}(t-1), \sum R S^{\phi, \zeta_{1}}(t-1)\right)-S P_{0}^{\zeta_{1}}(t)
$$

where $S P^{\zeta_{1}}(t)$ is the system performance of multi-layer network with restoration starting at $t$, $S P_{0}^{\zeta_{1}}(t)$ is the system performance of multi-layer network without restorations at $t$, $\sum S P^{\phi, \zeta_{1}}(t-1)$ is the integration of all single layer networks performance at $t-1$, $\sum R S^{\phi, \zeta_{1}}(t-1)$ is combined recovery strategies of all infrastructure types (layers) at $t-1$.

\subsubsection{Rapidity}

Rapidity refers to the capacity to meet priorities and achieve goals in a timely manner in order to minimize losses and avoid future infrastructure system disruptions. Duration of system recovery to normal operational levels is always used as a measure to evaluate system resilience, and can be seen as the main figure-of-merit to evaluate proactive recovery capacity. In this research, the duration of system recovery is used to describe rapidity, repressed as:

$$
\begin{aligned}
& R_{R a p}^{\phi, \zeta_{1}}=t_{1 R E}^{\phi}-t_{1 O}^{\phi} \quad \text { or } \\
& R_{R a p}^{\phi, \zeta_{1}}=t_{2 O}^{\phi}-t_{1 O}^{\phi}
\end{aligned}
$$

where $t_{1 R E}^{\phi}$ is the time when infrastructure network $\phi$ recovers to the operational level equal to the one before the disturbance event $\zeta_{1}, t_{1 O}^{\phi}$ is the occurrence time of the disturbance $\zeta_{1} \cdot t_{2 O}^{\phi}$ is the occurrence time of the following disturbance $\zeta_{2}$. Equation (10) is used in the situations with one disturbance event and Equation (11) in the case of multiple sequential disturbances.

For a multilayer infrastructure network, rapidity is computed as the longest duration of disturbance impacts of all the individual infrastructure network layers due to the specific disturbance $\zeta_{1}$, which is shown in Fig. 4 and represented as:

$$
R_{\text {Rap }}^{\zeta_{1}}=\max \left\{R_{\text {Rap }}^{\phi, \zeta_{1}}\right\}
$$

\subsection{Dynamic Infrastructure System Resilience Metric}

System performance and its adaptive capacity represent dynamic system behavior in response to system disturbance and the application of various adaptation measures. The original 


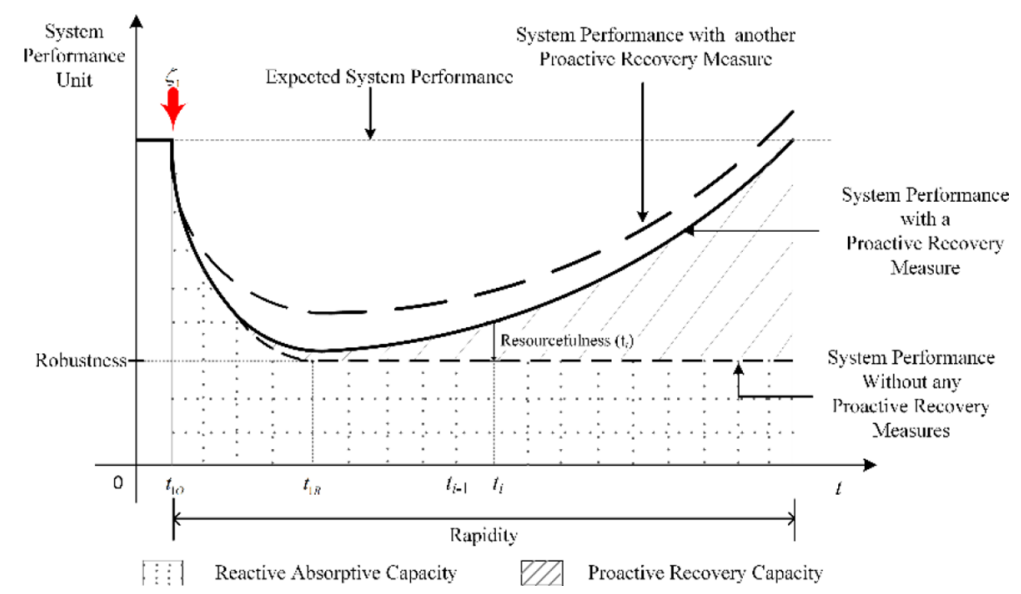

Figure 4: Typical performance process of an infrastructure system.

Space-Time Dynamic Resilience Measure developed by Simonovic and Peck [16] is adapted in this research to complex network infrastructure systems. It quantifies resilience as the difference between the area under the expected system performance and the actual system performance (dotted shaded area in Fig. 4). The introduction of system adaptation measures provides for the increase in system resilience (line shaded area in Fig. 4), where the system performance without adaptation measures is shown by the grey dashed line and with adaptation measures by the full black line. The adaptive capacity can be achieved by: proactive adjustment measures and reactive recovery measures.

\subsubsection{Proactive absorptive capacity metric}

With the three features introduced earlier (robustness, resourcefulness and rapidity), the proactive absorptive capacity of an individual infrastructure network subject to a disturbance $\zeta_{1}$ (represented as the dotted shaded area in Fig. 4) can be calculated as:

$$
\rho_{P A}^{\phi, \zeta_{1}}(t)=\frac{\int_{t_{10}}^{t} S P_{0}^{\phi, \zeta_{1}}(t) d t}{1 \times t}=\frac{\int_{t_{10}}^{t_{1 R}} S P_{0}^{\phi, \zeta_{1}}(t) d t+\int_{t_{10}}^{t_{1, R}^{\phi}} R_{R o b}^{\phi, \zeta_{1}}\left(t_{1 R}^{\phi}\right) d t}{1 \times t}, t \leq t_{1 R E}^{\phi}
$$

where $S P_{0}^{\phi, \zeta_{1}}(t)$ is the system performance of network $\phi$ after disturbance $\zeta_{1}$ without restoration strategy, which is calculated as the ratio of functional infrastructure network elements to total number of elements of network $\phi$ at $t$. And 1 in the denominator refers to the undisturbed system performance. After $t_{1 R}^{\phi}, S P_{0}^{\phi, \zeta_{1}}(t)$ would be stable and equal to $R_{R o b}^{\phi, \zeta_{1}}\left(t_{1 R}^{\phi}\right)$, which is the robustness of network $\phi$ after disturbance $\zeta_{1}$. For a multilayer infrastructure network, the proactive absorptive capacity metric can be described as:

$$
\rho_{P A}^{\zeta_{1}}(t)=\frac{\int_{t_{10}}^{t} \int_{\phi} S P_{0}^{\phi, \zeta_{1}}(t) d t}{1 \times t}=\frac{\int_{t_{10}}^{t_{1 R}} \int_{\phi} S P_{0}^{\zeta_{1}}(t) d t+\int_{t_{1 O}}^{t_{1 R}} \int_{\phi} R_{R o b}^{\zeta_{1}}\left(t_{1 R}\right) d t}{1 \times t}, t \leq t_{1 R E}
$$

The Proactive absorptive capacity of a multilayer infrastructure network could also be improved with the increase in its robustness, and be reduced with the decrease in its robustness. 


\subsubsection{Reactive recovery capacity metric}

Reactive recovery capacity of an individual infrastructure network subject to a disturbance $\zeta_{1}$ (illustrated as the line shaded area in Fig. 4) can be calculated as:

$$
\rho_{R R}^{\phi, \zeta_{1}}(t)=\frac{\int_{t_{10}}^{t} R_{R e s}^{\phi, \zeta_{1}}(t) d t}{1 \times t}, t \leq t_{1 R E}^{\phi}
$$

For a multilayer infrastructure network, the reactive recovery capacity metric is:

$$
\rho_{R R}^{\zeta_{1}}(t)=\frac{\int_{t_{10}}^{t} \int_{\phi} R_{\text {Res }}^{\zeta_{1}}(t) d t}{1 \times t}, t \leq t_{1 R E}
$$

\subsubsection{Resilience metric}

The resilience metric of a single layer infrastructure network $\phi$ under infrastructure network disturbance $\zeta_{1}$ can be now determined as:

$$
\begin{aligned}
r^{\phi, \zeta_{1}}(t) & =\rho_{P A}^{\phi, \zeta_{1}}(t)+\rho_{R R}^{\phi, \zeta_{1}}(t)=\frac{\int_{t_{1 O}}^{t} S P_{0}^{\phi, \zeta_{1}}(t) d t}{1 \times t}+\frac{\int_{t_{1 O}}^{t} R_{R e s}^{\phi, \zeta_{1}}(t) d t}{1 \times t}, t \leq t_{1 R E}^{\phi} \\
= & \frac{\int_{t_{1 O}}^{t_{1 R}} S P_{0}^{\phi, \zeta_{1}}(t) d t+\int_{t_{1 O}}^{t_{1 R}} R_{R o b}^{\phi, \zeta_{1}}\left(t_{1 R}\right) d t+\int_{t_{1 O}}^{t} R_{R e s}^{\phi, \zeta_{1}}(t) d t}{1 \times t}
\end{aligned}
$$

The resilience of the multilayer infrastructure network under infrastructure network disturbance $\zeta_{1}$ can be now determined as:

$$
\begin{aligned}
r^{\zeta_{1}}(t)= & \rho_{P A}^{\zeta_{1}}(t)+\rho_{R R}^{\zeta_{1}}(t)=\frac{\int_{\phi} \int_{t_{1 O}}^{t} S P_{0}^{\phi, \zeta_{1}}(t) d t}{1 \times t}+\frac{\int_{\phi} \int_{t_{10}}^{t} R_{R e s}^{\zeta_{1}}(t) d t}{1 \times t} \\
= & \frac{\int_{t_{10}}^{t_{1}} \int_{\phi} S P_{0}^{\phi, \zeta_{1}}(t) d t+\int_{t_{10} O}^{t_{1 R}} \int_{\phi} R_{R o b}^{\phi, \zeta_{1}}\left(t_{1 R}\right) d t+\int_{t_{10}}^{t} \int_{\phi} R_{R e s}^{\phi, \zeta_{1}}(t) d t}{1 \times t}, t \leq t_{1 R E}
\end{aligned}
$$

The resilience metrics in Equations (17) and (18) are derived for a single disturbance. Under a sequence of disturbances $\zeta_{1}, \zeta_{2}, \ldots \zeta_{d}$, single layer and multilayer infrastructure system resilience is the integral of the resilience under all single disturbances, which can be represented as $r^{\phi ; \zeta_{1}, \zeta_{2} \ldots \zeta_{d}}(t)$ and $r^{\zeta_{1}, \zeta_{2} \ldots \zeta_{d}}(t)$, and calculated as follows:

$$
\begin{gathered}
r^{\phi ; \zeta_{1}, \zeta_{2} \ldots \zeta_{d}}(t)=\rho_{P A}^{\phi ; \zeta_{1}, \zeta_{2} \ldots \zeta_{d}}(t)+\rho_{R R}^{\phi ; \zeta_{1}, \zeta_{2} \ldots \zeta_{d}}(t)=\frac{\int_{t_{10}}^{t} \int_{\zeta} S P_{0}^{\zeta}(t) d t}{1 \times t}+\frac{\int_{t_{10}}^{t} \int_{\zeta} R_{R e s}^{\zeta}(t) d t}{1 \times t}, t \leq t_{d R E}^{\phi} \\
r^{\zeta_{1}, \zeta_{2} \ldots \zeta_{d}}(t)=\rho_{P A}^{\zeta_{1}, \zeta_{2} \ldots \zeta_{d}}(t)+\rho_{R R}^{\zeta_{1}, \zeta_{2} \ldots \zeta_{d}}(t)=\frac{\int_{t_{10}}^{t} \int_{\zeta} \int_{\phi} S P_{0}^{\phi, \zeta}(t) d t}{1 \times t}+\frac{\int_{t_{10}}^{t} \int_{\zeta} \int_{\phi} R_{R e s}^{\phi, \zeta}(t) d t}{1 \times t}, t \leq t_{d R E}
\end{gathered}
$$

It's worth noting that the robustness under subsequent disturbances is always lower than that of the previous disturbance. This is because the time between sequential disturbances is always shorter than the time needed for the recovery to undisturbed level.

The resilience model developed in this work could be extended for the spatial distribution of disturbances. First, a disturbance is represented as the removal of functioning elements of 
the network. As natural disasters, such as severe weather conditions, earthquakes, and/or floods always strike geographically confined areas, the cell space method should be investigated. Second, system robustness to a specific disaster can be different as the structure of the infrastructure system changes. The proactive adaptive capacity changes accordingly. Third, the reactive recovery capacity can also be improved through resourcefulness and rapidity. Both of them are determined by the implementation of the adaptation/restoration strategy, which is the focus of single layer infrastructure network resilience. At last, duration of infrastructure malfunction does not only depend on the repair time, but also the buffering time $T_{B}$ and the state of dependent infrastructure systems. So, infrastructure system resilience analysis needs systematic understanding of internal infrastructure features, external disturbance attributes and overall integration mechnism.

\section{DISCUSSION}

Resilience is presented as an efficient approach for the management of infrastructure systems. This paper establishes a multilayer infrastructure system resilience model based on the Space-Time Dynamic Resilience Measure of Simonovic and Peck (2013) with consideration of infrastructure interdependences. By considering system performance, its adaptation capacity and consequences of specific restoration strategies, the resilience is represented as a dynamic measure to be implemented using system simulation.

Infrastructure system resilience takes advantage of the systems approach. As infrastructure systems mutually interact, understanding of system interdependences is essential for infrastructure system resilience analysis. This paper presents transformation of macro-perspective interdependences of infrastructure systems into micro dependence patterns. It integrates them through the application of system dynamics simulation analysis. The proposed resilience metric could be used for evaluation of different types of infrastructure systems with cascading failures or other high-order impacts. The presented model could be a generic framework or a methodology for the resilience analysis of systems-of-systems.

Finally, an actual infrastructure system is likely to be more complex. Integrating the consequences of disaster uncertainty into an infrastructure system evolution is another potential extension of the model to be considered in future work. The future research will include the case studies using a real infrastructure system data and disaster impacts.

\section{ACKNOWLEDGEMENTS}

We are grateful to the Natural Sciences and Engineering Research Council (NSERC) of Canada, and Chaucer plc (in cooperation with the Institute for Catastrophic Loss Reduction - ICLR) for their funding of this research.

\section{REFERENCES}

[1] Ouyang, M., Review on modeling and simulation of interdependent critical infrastructure systems. Reliability Engineering \& System Safety, 121, pp. 43-60, 2014. https://doi.org/10.1016/j.ress.2013.06.040

[2] Cutter, S.L., Barnes L., Berry M., Burton C., Evans E., Tate E. \& Webb J., A placebased model for understanding community resilience to natural disasters. Global Environmental Change, 18(4), pp. 598-606, 2008. https://doi.org/10.1016/j.gloenvcha.2008.07.013

[3] U S. Department of Homeland Security, National Infrastructure protection plan, 2013. 
[4] Francis, R. \& Bekera, B., A metric and frameworks for resilience analysis of engineered and infrastructure systems. Reliability Engineering \& System Safety, 121, pp. 90-103, 2014. https://doi.org/10.1016/j.ress.2013.07.004

[5] Bruneau, M., Chang, S.E., Eguchi, R.T, Lee, G.C., O'Rourke, T.D., Reinhorn, A.M., Shinozuka, M., Tierney, K., Wallace, W.A. \& von Winterfeldt, D., A framework to quantitatively assess and enhance the seismic resilience of communities. Earthquake Spectra, 19, pp. 733-752, 2003.

https://doi.org/10.1193/1.1623497

[6] Li, Y. \& Lence, B.J., Estimating resilience for water resources systems. Water Resources Research, 43(7), 2007. https://doi.org/10.1029/2006wr005636

[7] Maliszewski, P.J. \& Perrings, C., Factors in the resilience of electrical power distribution infrastructures. Applied Geography, 32(2), pp. 668-679, 2012. https://doi.org/10.1016/j.apgeog.2011.08.001

[8] Omer, M., Nilchiani, R. \& Mostashari, A., Measuring the resilience of the trans-oceanic telecommunication cable system. IEEE Systems Journal, 3(3), pp. 295-303, 2009. https://doi.org/10.1109/jsyst.2009.2022570

[9] D'Lima, M. \& Medda, F., A new measure of resilience: an application to the London underground. Transportation Research Part A: Policy and Practice, 81, pp. 35-46, 2015. https://doi.org/10.1016/j.tra.2015.05.017

[10] Golara, A. \& Esmaeily, A., Quantification and enhancement of the resilience of infrastructure networks. Journal of Pipeline Systems Engineering \& Practice, 8(1), p. 0406013, 2017. https://doi.org/10.1061/(asce)ps.1949-1204.0000250

[11] Zio, E. \& Sansavini, G., Vulnerability of smart grids with variable generation and consumption: a system of systems perspective. IEEE Transactions on Systems Man \& Cybernetics Part B, 43(3), pp. 477-487, 2013.

https://doi.org/10.1109/tsmca.2012.2207106

[12] Cagno, E., Ambroggi, M.D., Grande, O. \& Trucco, P., Risk analysis of underground infrastructures in urban areas. Reliability Engineering \& System Safety, 96(1), pp. 139-148, 2011. https://doi.org/10.1016/j.ress.2010.07.011

[13] Johansson, J. \& Hassel, H., An approach for modelling interdependent infrastructures in the context of vulnerability analysis. Reliability Engineering \& System Safety, 95(12), pp. 1335-1344, 2010.

https://doi.org/10.1016/j.ress.2010.06.010

[14] Filippini, R. \& Silva, A., A modeling framework for the resilience analysis of networked systems-of-systems based on functional dependencies. Reliability Engineering \& System Safety, 125, pp. 82-91, 2014. https://doi.org/10.1016/j.ress.2013.09.010

[15] Ouyang, M. \& Dueñas-Osorio, L. Time-dependent resilience assessment and improvement of urban infrastructure systems. Chaos: An Interdisciplinary Journal of Nonlinear Science, 22(3), p. 033122, 2012.

https://doi.org/10.1063/1.4737204 
[16] Simonovic, S.P. \& Peck, A., Dynamic resilience to climate change caused natural disasters in coastal megacities quantification framework. British Journal of Environment and Climate Change, 3(3), pp. 378-401, 2013. https://doi.org/10.9734/bjecc/2013/2504

[17] Cavallaro, M., Asprone, D., Latora, V., Manfredi, G. \& Nicosia, V., Assessment of urban ecosystem resilience through hybrid social-physical complex networks. Computer-Aided Civil and Infrastructure Engineering, 29(8), pp. 608-625, 2014. https://doi.org/10.1111/mice.12080

[18] Shuang, Q, Zhang, M. \& Yuan, Y., Node vulnerability of water distribution networks under cascading failures. Reliability Engineering \& System Safety, 124, pp. 132-141, 2014. https://doi.org/10.1016/j.ress.2013.12.002

[19] Albert, R., Albert, I. \& Nakarado, G.L., Structural vulnerability of the North American power grid. Physical Review E, 69(2), pp. 025103, 2004. https://doi.org/10.1103/physreve.69.025103

[20] Dudenhoeffer, D.D., Permann M.R. \& Manic M., CIMS: A framework for infrastructure interdependency modeling and analysis. Proceedings of the 38th conference on Winter simulation. Winter Simulation Conference, pp. 478-485, 2006. 